VOL. $2(1970), 223-228$.

\title{
An asymptotic result for a subcritical branching process with immigration
}

\author{
A. G. Pakes
}

Under weak hypotheses, the geometric ergodicity of the states of a subcritical branching process with immigration is demonstrated by obtaining an asymptotic representation of the transition probabilities.

Consider a branching process in which each individual reproduces independently of all others and has probability $a_{j}(j=0,1, \ldots)$ of giving rise to $j$ progeny in the following generation, and in which there is an independent immigration component where, with probability $b_{j}$ $(j=0,1, \ldots), j$ individuals enter the population at each generation. Letting $x_{n}(n=0,1, \ldots)$ be the population size of the $n$-th generation, it is well known [1], [2] that $\left\{x_{n}\right\}$ defines a Markov chain on the non-negative integers. Moreover, if $p_{i j}^{(n)}(i, j, n=0,1, \ldots)$ represents the n-step transition probabilities of this Markov chain, then

$$
P_{i}^{(n)}(x)=\sum_{j=0}^{\infty} p_{i j}^{(n)} x^{j}=\left(B\left(A_{n}(x)\right)\right)^{i} \prod_{m=0}^{n-1} B\left(A_{m}(x)\right)
$$

where, if $|x| \leq 1, A(x)=\sum_{j=0}^{\infty} a_{j} \cdot x^{j}, B(x)=\sum_{j=0}^{\infty} b_{j} x^{j}, A_{0}(x)=x$ and $A_{n+1}(x)=A\left(A_{n}(x)\right), \quad(n=0,1, \ldots)$.

Received 9 December 1969. This work was carried out during the tenure of a Department of Supply Postgraduate Studentship. 
If $0<a_{0}, b_{0}<1,0<a_{0}+a_{1}<1$ and $\alpha=A^{\prime}(1-)<1$, then the condition $\sum_{j=1}^{\infty} b_{j} \log j<\infty$ is necessary and sufficient for the existence of a limiting distribution, $\left\{\pi_{j}\right\}$, of the Markov chain and

$$
\Pi(x)=\sum_{j=0}^{\infty} \pi_{j} x^{j}=\prod_{m=0}^{\infty} B\left(A_{m}(x)\right) \quad(|x| \leq 1),
$$

see $[1],[2]$ and $[6]$.

In [5] the author studied the rate of convergence of $p_{i j}^{(n)}$ to its limit $\pi_{j}$ and it was shown that if $\beta=B^{\prime}(1-)<\infty, p_{i j}^{(n)}-\pi_{j}=O\left(\alpha^{n}\right)$, showing that all the states of the Markov chain are geometrically ergodic. More precisely, if also $\sum_{j=1}^{\infty} a_{j} j \log j<\infty$ then $p_{i j}^{(n)}-\pi_{j}=\alpha^{n}\left(M_{i j}+o(1)\right)$ and the exact form of $M_{i j}$ was found, but if $\sum_{j=1}^{\infty} a_{j} j \log j=\infty$ then $p_{i j}^{(n)}-\pi_{j}=o\left(\alpha^{n}\right)$

The purpose of this note is to show that qualitatively similar results obtain if we relax the requirement that the immigration distribution have a finite mean, but we shall assume that $B(x)$ is given in terms of a slowly varying function. We now prove the following theorem.

THEOREM. Let $0<a_{0}, b_{0}<1,0<a_{0}+a_{1}<1, \alpha<1$ and $\sum_{j=1}^{\infty} a_{j} j \log j<\infty$ for a branching process with immigration. If

$$
B(1-x)=1-x^{\delta} L(x) \quad(0<\delta \leq 1)
$$

where $L(x)$ is slowly varying at the origin, then

$$
P_{i}^{(n)}(x)=\pi(x)+\alpha^{n \delta} L\left(\alpha^{n}\right)(H(s)+o(1)) \quad(i=0,|x|<1)
$$

where $H(x)=\Pi(x)(1-G(x))^{\delta} / c^{\delta}\left(1-\alpha^{\delta}\right), c=G^{\prime}(1-)$ and $G(x)$ is a 
probability generating function satisfying the functional equation

$$
1-G(A(x))=\alpha(1-G(x)) \text {. }
$$

The n-step transition probabilities have the form

$$
p_{i j}^{(n)}=\pi_{j}+\alpha^{n \delta} L\left(\alpha^{n}\right)\left(h_{j}+o(1)\right) \quad(i=0, j=0,1, \ldots)
$$

where $H(x)=\sum_{j=0}^{\infty} h_{j} x^{j}$. If $0<\delta<1$ or $\delta=1$ and $L(x) \rightarrow \infty$,

$(x+0)$ then equations (4) and (5) also hold for $i=1,2, \ldots$.

Proof. The condition for the existence of a limiting distribution,

$\sum_{j=1}^{\infty} b_{j} \log j<\infty$, is equivalent to the condition $\int_{0}^{\gamma}(1-B(1-x)) / x d x<\infty$, $(0<\gamma \leq 1)$, and it follows from equation (3) that this latter condition is indeed satisfied on recalling that for a slowly varying function

$$
x^{\varepsilon} L(x) \rightarrow 0 \quad(x+0 ; \varepsilon>0) .
$$

Considering the case $i=0$, it follows from equations (1) and (2) that

$$
P_{0}^{(n)}(x)-\Pi(x)=P_{0}^{(n)}(x)\left(1-\prod_{m=n}^{\infty} B\left(A_{m}(x)\right)\right)
$$

The first member on the right converges to $\Pi(x)$ and it is not hard to show that for the second member,

$$
1-\prod_{m=n}^{\infty} B\left(A_{m}(x)\right)=\sum_{m=n}^{\infty}\left(1-B\left(A_{m}(x)\right)\right)+R^{(n)}(x)
$$

where

(9) $\quad-\left[\sum_{m=n}^{\infty}\left(1-B\left(A_{m}(0)\right)\right)\right]^{2} / 2 \leq R^{(n)}(x) \leq \sum_{m=n}^{\infty}\left(1-B\left(A_{m}(0)\right)\right)^{2} / B\left(A_{m}(0)\right)$

and we have assumed that $0 \leq x<1$. We shall now obtain an asymptotic estimate of the series in (8). Using equation (3) we have

$$
\sum_{m=n}^{\infty} \frac{1-B\left(A_{m}(x)\right)}{\alpha^{n \delta} L\left(\alpha^{n}\right)}=\sum_{m=0}^{\infty}\left(\frac{1-A_{m+n}(x)}{\alpha^{n+m}}\right)^{\delta} \cdot \frac{L\left(1-A_{m+n}(x)\right)}{L\left(\alpha^{n+m}\right)} \cdot \frac{L\left(\alpha^{n+m}\right)}{L\left(\alpha^{n}\right)} \alpha^{m \delta} .
$$


Since $\sum_{j=1}^{\infty} a_{j} j \log j<\infty$, we can combine the refined forms of Yaglom's and Kolmogorov's limit theorems (see [3]) to obtain

$$
1-A_{n}(x)=\alpha^{n}[(1-G(x)) / c+o(1)]
$$

where $G(x)$ and $c$ are as given in the statement of the theorem. Thus, for each $x \in(0,1)$, the first factor of the terms of the series on the right hand side of (10) is bounded, and the boundedness of the second factor follows from $(11)$ and the fact that $L(t y) / L(y) \rightarrow 0,(y+0)$, uniformly for $0<u<t<U<\infty$; see [4]. Finally use of (7) makes it clear that the dominated convergence theorem is applicable to equation (10) thus yielding

$$
\sum_{m=n}^{\infty}\left[1-B\left(A_{m}(x)\right)\right]=\alpha^{n \delta} L\left(\alpha^{n}\right)\left[(1-G(x))^{\delta} / c^{\delta}\left(1-\alpha^{\delta}\right)+o(1)\right] \quad(0 \leq x<1) \text {. }
$$

It is apparent from the working above and Kolmogorov's limit theorem that

$$
1-B\left(A_{n}(0)\right)=\alpha^{n \delta} L\left(\alpha^{n}\right)(1 / c+o(1)) .
$$

Using this result and the well known fact that $A_{n}(0) \uparrow 1,(n \rightarrow \infty)$, it is not hard to show from equation (9) that $R^{(n)}(x)=0\left[\left(\alpha^{n} L\left(\alpha^{n}\right)\right)^{2}\right]$. Hence equation (4) is proven in the case $0 \leq x<1$.

$$
\text { On observing that }\left|1-B\left(A_{n}(x)\right)\right| \leq 2\left|1-B\left(A_{n}(0)\right)\right|, \quad(|x| \leq 1),
$$

and using the inequality $\left|1-\prod_{m=n}^{\infty} B\left(A_{n}(x)\right)\right| \leq 1-\prod_{m=n}^{\infty}\left(1-\left|1-B\left(A_{n}(x)\right)\right|\right)$, it is clear from the above that $\left[P_{0}^{(n)}(x)-\Pi(x)\right] / \alpha^{n} L\left(\alpha^{n}\right)$ is uniformly bounded in $|x| \leq 1$ and so, by Vitali's theorem, this sequence of functions converges uniformly in compact subsets of the open unit disc to $H(x)$, thus completing the proof of equations (4) and (6).

Letting now $i>0$, we have 


$$
\frac{P_{i}^{(n)}(x)-\Pi(x)}{\alpha^{n \delta} L\left(\alpha^{n}\right)}=\frac{P_{0}^{(n)}(x)-\Pi(x)}{\alpha^{n \delta} L\left(\alpha^{n}\right)}-\frac{1-A_{n}(x)}{\alpha^{n}} \cdot \frac{P_{0}^{(n)}(x) \sum_{k=0}^{i-1}\left[A_{n}(x)\right]^{k}}{\alpha^{-n(1-\delta)} L\left(\alpha^{n}\right)}
$$

and $\alpha^{-n(1-\delta)} L\left(\alpha^{n}\right) \rightarrow \infty$ under either of the two conditions given in the last assertion of the theorem. Thus the left hand side of the expression above converges uniformly to $H(x)$ in compact subsets of the open unit disc, thus completing the proof.

In view of equation (7), it is clear from (6) that the states of $\left\{X_{n}\right\}$ are geometrically ergodic, and if $h_{j}>0$, the convergence

parameter can be taken as any number in the interval $\left(0, \alpha^{\delta}\right)$. Observe that, under the conditions given in the last assertion of the theorem, all moments of the limiting distribution are infinite.

Under the condition $\sum_{j=1}^{\infty} a_{j}{ }^{j l o g j}=\infty$, it is shown in [3] that $c=\infty$. Referring to equation (9), we see that the first factor of the series on the right can be dominated by arbitrary $\varepsilon>0$ if $n$ is large enough. Thus we have the following.

COROLLARY. If alz the conditions of the theorem hold, except that $\sum_{j=1}^{\infty} a_{j} j \log j=\infty$, then we have

$$
p_{i j}^{(n)}=\pi_{j}+L\left(\alpha^{n}\right) r_{i j}^{(n)}
$$

where $r_{i j}^{(n)}=o\left(\alpha^{n \delta}\right), \quad(n \rightarrow \infty)$.

Once again we see that the states are geometrically ergodic.

\section{References}

[1] C.R. Heathcote, "A branching process allowing immigration", J. Roy. Statist. Soc. Ser. B 27 (1965), 138-143.

[2] C.R. Heathcote, "Corrections and comments on the paper 'A branching 
process allowing immigration' ", J. Roy. Statist. Soc. Ser. B $28(1966), 213-217$.

[3] C.R. Heathcote, E. Seneta and D. Vere-Jones, "A refinement of two theorems in the theory of branching processes", (Russian summary), Teor. Verojatnost. i Pmimenen. 12 (1967), 341-346.

[4] J. Korevaar, T. van Aardenne-Ehrenfest and N. de Bruijn, "A note on slowly oscillating functions", Nieuw Arch. Wisk. (2) 23 (1949), $77-86$.

[5] A.G. Pakes, "Branching processes with immigration", submitted to J. Roy. Statist. Soc.

[6] E. Seneta, "Functional equations and the Galton-Watson process", Advances App Z. Prob. 1 (1969), 1-42.

Monash University,

clayton, Victoria. 